\title{
Correlation of Human Epidermal Growth Factor Receptor (Her2-neu) Marker with the Grades of Urothelial Carcinoma
}

\author{
Maryam Bibi ${ }^{1}$, Walayat Shah ${ }^{2}$, Muhammad Asif ${ }^{1}$, Maria Tasneem Khattak ${ }^{3}$, Aisha Jamil', Bushra Nasib ${ }^{2}$ \\ ABSTRACT
}

Background: Urothelial carcinoma is the common carcinoma of urothelium. Its incidence is high in developed countries but its occurrence is increasing in developing countries like Pakistan with mortality rate of 3.8/100,000 in males and 1/100,000 in females. HER2 is a member of the human epidermal growth factor receptor (HER/EGFR/ERBB) family. Amplification or over-expression of this oncogene has been shown to play an important role in the development and progression urothelial carcinoma.

Objective: To evaluate the relationship between HER2/NEU expression and grades of urothelial carcinoma bladder

Material and Methods: This was a cross-sectional descriptive study for the period of six months from January to June, 2017 conducted at Pathology section Rehman Medical Institute Peshawar. After approval from university Ethical board 63 cases were selected by purposive sampling.

The Tissue samples from diagnosed cases of urothelial carcinoma of urinary bladder were included.

Cases of urothelial carcinoma from other sites were excluded. Paraffin embedded tissue blocks were cut and sections stained by H\&E to evaluate histopathological features and tumor grades. Immunohistochemical expression of HER2 was determined applying standard techniques of immunohistochemistry.

Results: Out of total 63 cases, 47 were males and 16 were female with a mean age of 60.7 years (range 14-90 years). There were 38 High grade, 23 Low grade and 2 cases of PUNLMP. 36 cases were positive for HER2 expression in which 26(72.2\%) were High grade, $9(25 \%)$ of Low grade and $1(2.7 \%)$ of PUNLMP. High grade was more common in HER2 positive cases and this relationship was significant $(p<.05)$. Among positive 36 cases, 19 cases of +3 score were High grade, 05 in Low grade and 01 in PUNLMP while score 2+ was in 07 cases of High grade and 04 were in Low grade. The relationship of HER2 score was not significantly related to grade of tumor $(p>.05)$

Conclusion: HER2 is highly expressed in high grades of urothelial carcinoma bladder and this relationship between HER2 expression and tumor grades is statistically significant.

Keywords: HER2, Urothelial carcinoma, Grades, Immunohistochemistry

This article may be cited as: Bibi M, Shah W Asif M, Khattak MT, Jamil A, Nasib B. Correlation of Human Epidermal growth factor receptor (Her2-Neu) marker with the Grades of Urothelial Carcinoma. J Saidu Med Coll Swat 2021;11(1): 24-29. doi:10.5226/jsmc.2021.11.1.24-29

INTRODUCTION

Bladder carcinoma is the ninth most common cause of cancer worldwide with male to female ratio of 3.5:1 respectively. ${ }^{1}$ Morphologically speaking, urothelial carcinoma which arises in urothelial/transitional epithelium makes $90 \%$ of all bladder cancers. ${ }^{2}$ Pakistan carries a high burden of newly diagnosed cases of urothelial carcinoma with mortality rate of $3.8 / 100,000$ in males and $1 / 100,000$ in females. $^{1}$

Etiologic factors of bladder carcinoma include smoking, industrial carcinogens as aromatic amines and aniline dyes, radiations, medications containing phenacetin compound and some chemotherapeutic drugs as cyclophosphamide, and genetic polymorphism. Chronic cystitis with schistosoma is implicated in development of squamous cell carcinoma in African countries. ${ }^{3}$

1. Gajju Khan Medical College Swabi

2. Khyber Medical University Peshawar

3. Rehman College of Dentistry and Consultant Histopathologist RMI Peshawar

Dr. Muhammad Asif

Pathology Department Gajju Khan Medical College Swabi

Email: doctorasif66@gmail.com

Cell No: 03339669042
Urothelial carcinoma arises from the urothelial/transitional epithelium of urinary bladder. $70-80 \%$ bladder tumor are superficial confined to mucosa and have good prognosis. ${ }^{4}$ The resting $20-30 \%$ are invasive to muscles with worse outcome. ${ }^{5}$

Tumor Grade and stage are two important factors to determine prognosis, risk of recurrence and progression of disease. Molecular markers are involved in pathways of carcinogenesis and progression and their identification can aid into the prognosis. $^{6}$

Human Epidermal growth Factor Receptor 2/ HER2 /NEU is an oncogene related to a family of epidermal growth factor receptors. It is located on chromosome 17. These are type 1 transmembrane growth factor receptors which activate intracellular signaling pathways in response to external signals. ${ }^{7}$ HER2 overexpression and amplification is a common mechanism of oncogenesis in various tumors. Overexpression of HER2 is studied in invasive breast carcinoma, gastric carcinoma, ovarian, endometrial, esophageal and lung cancers. Urothelial carcinoma has a variable range of expression from $2-74 \%{ }^{8}$ which has a mixed association with prognosis of the carcinoma. It is

Received: January 06, 2019 Accepted: August 18, 2020 
related to aggressive behavior in some studies while other shows no association to prognosis. .,10 $^{2}$ HER2 targeted therapies have promising role in breast carcinoma. ${ }^{8}$ Immune therapy with antibody directed against HER2 can be a potential therapy in bladder carcinoma esp. in advanced cases.

This study aims to determine HER2 expression in urothelial carcinoma bladder and its association with tumor grade.

\section{MATERIALS AND METHODS}

This was cross sectional analytical study carried out at histopathology section of pathology laboratory at Rehman Medical Institute Peshawar. Non probability purposive sampling was done and using formula

$$
n=Z^{2} * 1-\alpha * \frac{\rho(1-\rho)}{d^{2}}
$$

Where $d=$ margin of error usually at $0.05, z=$ standard deviation usually at 1.96 corresponds to $95 \%, \alpha=0.05$ and $\rho=$ prevalence of disease which is $0.05(5 \%)^{11}$, the sample size was calculated as 63 .

Tissue specimens of 63 cases of urothelial carcinoma bladder were collected between January to June 2017. Institutional based Ethical clearance was received to conduct the study. Tissue blocks of all diagnosed cases were collected. Cases of bladder carcinoma other than Urothelial carcinoma were excluded. Urothelial carcinoma from other sites of urinary tract other than bladder was also not included.

Thin sections of 2-3 $\mu \mathrm{m}$ were cut from tissue blocks and slides prepared. Hematoxylin and Eosin staining was carried out according to standard protocol. ${ }^{12}$ Histomorphological features of carcinoma studied and tumor was graded according to World Health Organization (WHO) classification of tumors grading $2004 .{ }^{13}$

For immunohistochemistry, slides were first deparaffinized by heating in microwave and then putting in xylene for 05 minutes. Heat induced epitope retrieval method was used. Slides were put in EDTA solution and heated in microwave oven for 15-20 minutes at 140 'c. Peroxidase blocking reagent was applied for 05 minutes and then washed with Phosphate Buffer Saline. Next, Primary antibody (DAKO, polyclonal rabbit antiHuman c-erbB2 antibody) was put on and slides were incubated in humidified chamber for an hour. Secondary antibody using (Horseradish Peroxidase conjugated antibody, Envision FLEX/HRP DM822) was administered and again slides were incubated in humidified chamber for 30 minutes. Finally DAB reagent was applied until a homogeneous color obtained. Counterstaining was done with Hematoxylin solution. Slides were mounted with DPX medium, coverslips applied and labeled. ${ }^{14}$ HER2 expression was noted and scored according to American Society of Clinical Oncology scoring as following: ${ }^{15}$

Score 3+: Circumferential membrane staining that is complete, intense and in $>10 \%$ of tumor cells.

Score 2+: Circumferential membrane staining that is incomplete, weak/moderate and in $>10 \%$ of tumor cells or complete and circumferential membrane staining that is intense and within $<10 \%$ of tumor cells.

Score 1+: Incomplete membrane staining that is faint/barely perceptible and within $>10 \%$ of tumor cells.

Score 0: No staining is observed or incomplete membrane staining that is faint/barely perceptible and in $<10 \%$ of tumor cells.

Frequencies were calculated for variables and Chi-square test applied to determine association between variables. Data arranged and analyzed in SPSS (version 20.0 IBM). Results were plotted in tables. Immunohistochemistry staining and scores were shown in figures.

\section{RESULTS}

Total 63 cases of urothelial carcinoma were included in this study. Age and gender distribution among all cases is shown in Table No 1.

Table No. 1. Age and Sex distribution of cases

\begin{tabular}{|c|c|c|}
\hline Sex & No. (\%) & Mean age (range) years \\
\hline Males & $47(75 \%)$ & $63.12(20-92)$ \\
\hline Females & $16(25 \%)$ & $53.5(14-70)$ \\
\hline Total & $63(100)$ & $60.68(14-92)$ \\
\hline
\end{tabular}


Of total 63 cases, 38(60\%) were classified as high grade, $23(37 \%)$ as low grade carcinoma while 02 cases were of Papillary Urothelial Neoplasm of Low Malignant Potential (PUNLMP). Among 38 cases of high grade, $31(81.5 \%)$ were invasive and $2(5 \%)$ noninvasive cases. The low grade 23 cases had $13(61.9 \%)$ invasive whereas $6(26 \%)$ were noninvasive cases. 05 cases of high grade and 04 cases of low grade had no details about stage of tumor.

The positive and negative cases among different grades were as described in table 02 . High grade had increased number of HER2 positive cases as compared to low grade $(68.4 \%$ vs. $39 \%)$ and this was statistically proved as significant $(p=0.025$, $\mathrm{p}<0.05)$

Among the positive 36 cases, 25 (69\%) cases scored 3+ while 11(31\%) scored 2+ for HER2 expression. 21 cases (78\%) scored $1+$ and 06 cases $(22 \%)$ scored 0 for HER2 among negative 27 cases.ImmunohistochemicallyHER2 expression is shown in figure 01 . The distribution of HER2 scores in different grades is as given in table 03.

There was increased number of score $3+$ in high grade $(50 \%)$ as compared to low grade $(22 \%)$. Score2+ score was nearly same in high and low grades (18\% in high grade vs. $17 \%$ in low grade). Low grade had increased number of score 1+ cases as compared to high grade (12 cases in low grade vs. 8 in high grade) whereas score 0 was nearly same in high grade $4(11 \%)$ and low grade $2(9 \%)$. This association of grades and HER2 expression score was statistically proved insignificant as $p=0.06(p>0.05)$
Figure No. 1. IHC staining shows HER2 expression as membranous staining of cells (a) score $3+$ with complete, circumferential and intense membrane staining in $>10 \%$ of cells (b) score 2+with incomplete, circumferential and weak/moderate membrane staining in $>10 \%$ cells (c) score 1+ with incomplete, weak/faint and partial membrane staining in $>10 \%$ cells $(d)$ score 0 with no membrane staining of cells (400X).

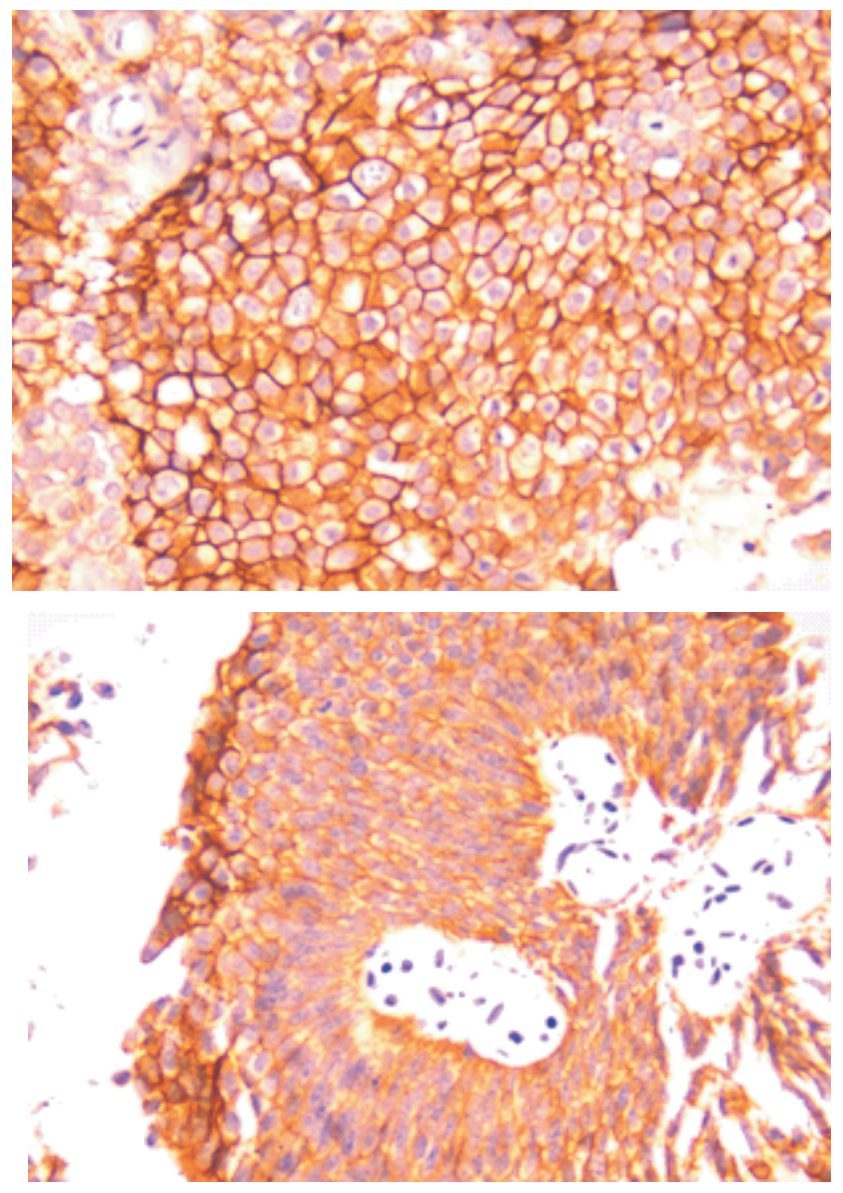

Table 2. Distribution of HER2 positive and negative cases in different grades

\begin{tabular}{|c|c|c|c|c|}
\hline Cases & Positive & Negative & Tota I & P value \\
\hline High grade & $26(68.4 \%)$ & $12(31.6 \%)$ & $38(60 \%)$ & \multirow{2}{*}{$\begin{array}{c}\mathrm{p}=.025 \\
(p<0.05)\end{array}$} \\
\cline { 1 - 3 } Low grade & $9(39 \%)$ & $14(60.9 \%)$ & $23(36.5 \%)$ & $2(3 \%)$ \\
\cline { 1 - 3 } PUNLMP & $1(50 \%)$ & $1(50 \%)$ & $63(100 \%)$ & \\
\hline Tota I & $36(57 \%)$ & $27(43 \%)$ & \\
\hline
\end{tabular}

Table 3. HER2 score expression among different grades of tumor

\begin{tabular}{|c|c|c|c|c|c|c|c|c|}
\hline \multirow{2}{*}{ Grade/score } & \multirow{2}{*}{$\begin{array}{l}\text { No. } \\
\text { (n) }\end{array}$} & \multicolumn{2}{|c|}{ Positive } & \multirow{2}{*}{ Tota I } & \multicolumn{2}{|c|}{ Negative } & \multirow{2}{*}{ Tota I } & \multirow[t]{2}{*}{ P value } \\
\hline & & +3 & +2 & & +1 & 0 & & \\
\hline High grade & 38 & $19(50 \%)$ & $7(18 \%)$ & 26 & $8(21 \%)$ & $4(11 \%)$ & 12 & \multirow{4}{*}{$\begin{array}{c}p=.06 \\
(p>0.05)\end{array}$} \\
\hline Low grade & 23 & $5(22 \%)$ & $4(17 \%)$ & 9 & $12(52 \%)$ & $2(9 \%)$ & 14 & \\
\hline PUNLMP & 2 & $1(50 \%)$ & - & 1 & $1(50 \%)$ & - & 1 & \\
\hline Tota I & 63 & $25(69 \%)$ & $11(31 \%)$ & 36 & $21(33 \%)$ & $6(10 \%)$ & 27 & \\
\hline
\end{tabular}



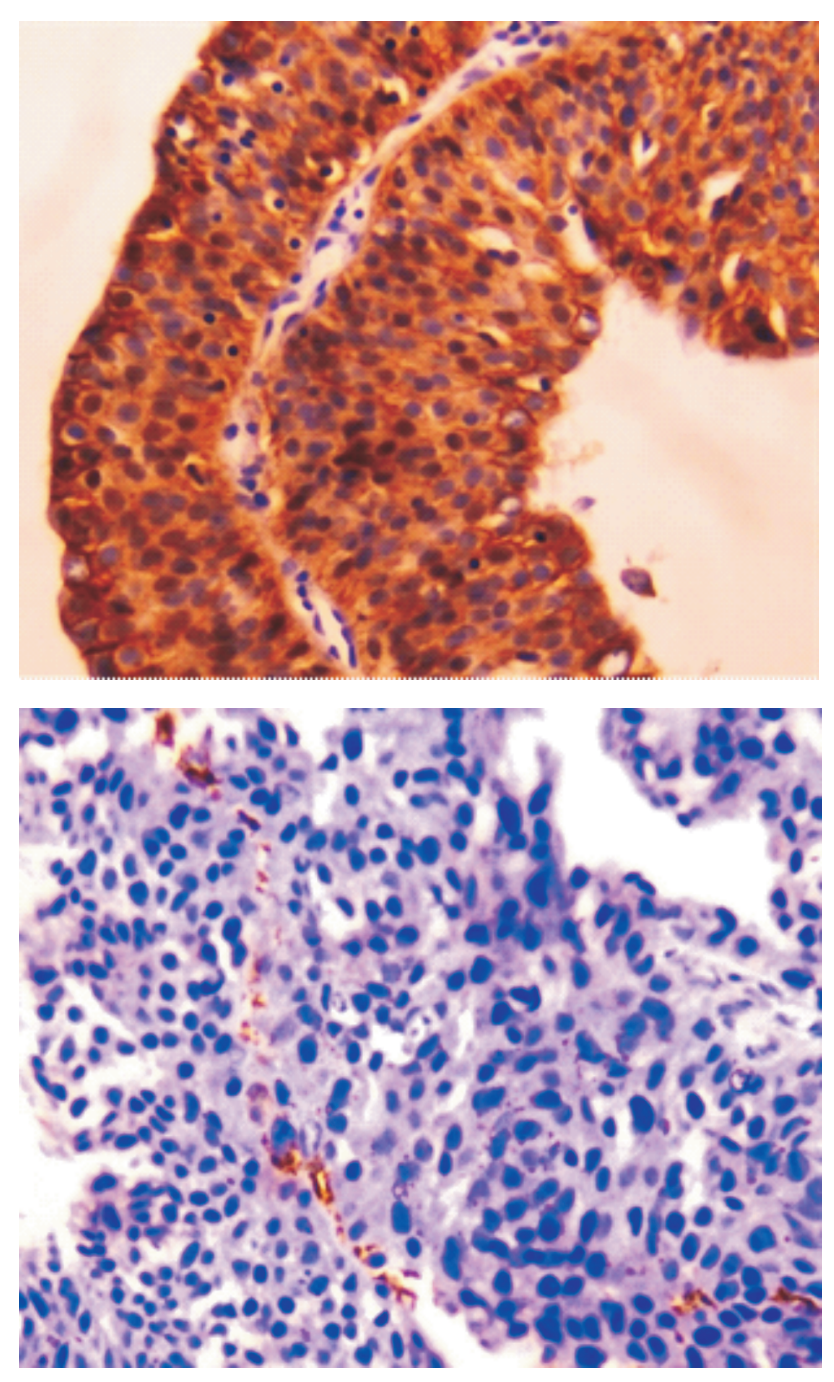

DISCUSSION

Human epidermal growth factor receptor

(HER2/neu) is a known biomarker used in breast cancer and therapy directed against it has marked effects in treatment. Its role as a prognostic marker in other non-breast cancers (esophageal and ovarian cancers) is also established and is targeted for treatment. ${ }^{9}$

HER2 expression in urothelial carcinoma of urinary bladder varies, ranging from $2-74 \%$ according to different studies. ${ }^{17}$. In bladder urothelial carcinoma, its role as a prognostic marker is debatable. ${ }^{18,19}$ In many studies, it is said to be related to high grade, aggressiveness and worse prognosis. ${ }^{18-24}$

In this study, Positive expression of HER2 was $57 \%$ which is close in range to that noted by Shawky et al with 53\% HER2 expression in invasive TCC bladder carcinoma cases. ${ }^{18} \mathrm{Khaled}$ El Ghani also noted $52 \%$ positive HER2 expression in their study. ${ }^{25}$
Positive expression was more common in high grade $(68.4 \%)$ than Low grade carcinoma (39\%) in this study and this association was statistically significant. This finding was also noted by Nour ElHuda in their study, with $62.8 \%$ positive expression in High grade and $11.8 \%$ in Low grade cases ${ }^{26}$ Nighat and colleagues noted $47.5 \%$ and $16.6 \%$ cases positive for HER2 in High and Low grades respectively. ${ }^{27}$ Khaled El Ghani found out HER2 expression in $66.6 \%$ High grade and $44.4 \%$ in Low grade cases but this relationship was insignificant. $^{25}$

High grade has high number of positive score 3+ $(50 \%)$ as compared to low grade $(22 \%)$ while score $2+$ was nearly same in both grades. Similar findings were noted by Khaled El Ghani. ${ }^{25}$ Nour El Huda et al had same expression of score $3+$ in high grade but there was no difference in positive scores in low grade (score $3+5.9 \%$, score $2+$ $5.9 \%$ ) in their study. ${ }^{26} \mathrm{Nejdadi}$ et al, in contrast, found more number of score2+ in low grade than high grade whereas score $3+$ was nearly same in both low and high grades. ${ }^{24}$

Invasive stage had more common positive expression (63.6\%) than noninvasive stage $(37.5 \%)$ where negative cases were more frequent though this was statistically insignificant. Nighat and colleagues also noted more positive HER2 expression in invasive stage than noninvasive stage. ${ }^{27}$ Tereihi et al, also noted HER2 highly expressed in invasive carcinomas while absent in superficial tumors. ${ }^{21}$ Nour el Huda and colleagues also studied similar findings. ${ }^{26}$

Though HER2 expression was significantly related to tumor grade yet the individual scores of HER2 expression had insignificant association with grades. This finding can be accurately verified by advanced techniques as FISH. Nejdadi and colleagues in their study noted concordance of results between IHC and BDISH for HER2 status where High grades and higher scores of HER2 had increased number of HER2 amplification. ${ }^{24}$ Further investigations by more advanced techniques as FISH or flow IHC are needed to verify HER2 status especially in those of equivocal cases. Stage of carcinoma was not studied in detail and the relationship with HER2 must be determined properly which is important in prognosis. Immune mediated treatments against HER2 are already working in breast carcinoma and this gives hope to urothelial carcinoma once HER2 status is established accurately. 


\section{CONCLUSION}

High grade urothelial carcinoma bladder has significant positive association with HER2 expression. The association between HER2 expression score and grades needs further studies and confirmation by Florescence in situ hybridization (FISH), especially in borderline cases of HER2 score 2+ where alternative confirmation is by FISH.

\section{REFERENCES}

1. International Agency for Research on Cancer and others. GLOBOCAN 2012: estimated cancer incidence, mortality and prevalence worldwide in 2012 [Online][accessed on 20 May 2019]. Available from: http://globocan.iarc.fr/pages/fact_sheets_population. aspx

2. Hall MC, Chang SS, Dalbagni G, Pruthi RS, Seigne JD, Skinner EC, et al. Guideline for the management of non muscle invasive bladder cancer (stages Ta, T1, and Tis): 2007 update. J Urol. 2007; 178(6):2314-30.

3. Murta-Nascimento C, Schmitz-Dräger BJ, Zeegers MP, Steineck G, Kogevinas M, Real FX, et al. Epidemiology of urinary bladder cancer: from tumor development to patient's death. World J Urol. 2007; 25(3):285-95.

4. Mansoor M, Ali S, Fasihuddin Q, Baloch MU. Superficial bladder tumours: recurrence and progression. J Coll Physicians Surg Pak. 2011; 21(3):157-60

5. Burger M, Catto JW, Dalbagni G, Grossman HB, Herr $H$, Karakiewicz $P$, et al. Epidemiology and risk factors of urothelial bladder cancer. Eur Urol. 2013; 63(2):234-41

6. Sharma S, Ksheersagar P, Sharma P. Diagnosis and treatment of bladder cancer. Am Fam Physician. 2009; 80(7):717-23.

7. Kausch I, Bohle A. Molecular aspects of bladder cancer: III. Prognostic markers of bladder cancer. Eur Urol. 2002; 41(1):15-29

8. Herter-Sprie GS, Greulich H, Wong KK. Activating Mutations in ERBB2 and Their Impact on Diagnostics and Treatment. Front Oncol. 2013; 3:86-93.

9. Iqbal N, Iqbal N. Human Epidermal Growth Factor Receptor 2 (HER2) in Cancers: Overexpression and Therapeutic Implications. MolBiollnt 2014; 2014:9-17

10. Gandour-Edwards R, Lara PN, Folkins AK, LaSalle JM, Beckett L, Li Y, et al,. Does HER2/neu expression provide prognostic information in patients with advanced urothelial carcinoma? Cancer. 2002; 95(5):1009-15.

11. Khan MK, Ahmed I, Raza SJ. Factors affecting recurrence and progression of high grade noninvasive bladder cancer treated by intravesical BCG. Pak J Med Sci 2014:30(2):326-30

12. Olsson H, Fyhr I-M, Hultman P, Jahnson S. HER2 status in primary stage T1 urothelial cell carcinoma of the urinary bladder. Scand J UrolNephrol. 2012; 46(2):10-27
13. Feldma AT, Wolfe D. Tissue Processing and Hematoxylin and Eosin Staining. In: Christina E. Day, editor. Histopathology: Methods in Molecular Biology (Methods and Protocols). DOI: http://doi.org/10.1007/978-1-4939-1050-2_3: Humana press, New York, NY; 2014. p. 31-43.

14. Montironi R, Lopez-Beltran A. The 2004 WHO classification of bladder tumors: a summary and commentary. International journal of surgical pathology. Sage Publications Sage CA: Thousand Oaks, CA; 2005;13(2):143-53

15. Ramos-Vara JA. Principles and methods of immunohistochemistry. Methods Mol Biol. 2011;691:83-96

16. Antonio C. Wolff, M. Elizabeth H. Hammond, Kimberly H. Allison, David G. Hicks, Mitch Dowsett, Lisa M. McShane, et al., Recommendations for Human Epidermal Growth Factor Receptor 2 Testing in Breast Cancer: American Society of Clinical Oncology/College of American Pathologists Clinical Practice Guideline Update. Journal Of Clinical Oncology. 2013; 31(31): 3997-4014

17. Krüger $S$, Weitsch $G$, Büttner $H$, Matthiensen $A$, B?hmer T, Marquardt T, et al,. HER2 overexpression in muscle-invasive urothelial carcinoma of the bladder: Prognostic implications. Int J Cancer. 2002; 102(5):514-8.

18. Alexa A, Baderca F, Zahoi DE, Lighezan R, Izvernariu D, Raica M. Clinical significance of Her2/neu overexpression in urothelial carcinomas. Rom J MorpholEmbryol. 2010; 51(2):277-82.

19. Kumar S, Prajapati O, Vaiphei K, Parmar KM, Sriharsha AS, Singh SK. Human epidermal growth factor receptor $2 /$ neu overexpression in urothelial carcinoma of the bladder and its prognostic signifcance: Is it worth hype?. South Asian J Cancer 2015; 4:115-7.

20. Shawky AE-A, Elosaily G, Al-Matubsi H, Farahat A, others. Her-2/Neu Overexpression in Invasive Bladder Carcinoma among a Cohort of Egyptian Patients. World J Nephrol Urol. 2013; 2(2):705-13

21. Al-Tereihi RG Kerbel HA, Saheb RH, As'ad A. Overexpression of HER-2/ neu receptor protein in Urinary Bladder Carcinoma, An Immunohistochemical Study. J Fac Med Baghdad. 2011; 53(2):175-79.

22. Chen $\mathrm{PCH}, \mathrm{Yu} \mathrm{HJ}$, Chang $\mathrm{YH}$, Pan CC. Her2 amplification distinguishes a subset of non-muscleinvasive bladder cancers with a high risk of progression. J ClinPathol. 2013; 66(2):113-9

23. El Ochi MR, Oukabli M, Bouaiti E, Chahdi H, Boudhas A Allaoui M, Ameur A, et al. Expression of human epidermal growth factor receptor 2 in bladderurothelial carcinoma.BMC Clinical Pathology. 2017; 17(1):3-11.

24. Nedjadi T, Al-Maghrabi J, Assidi M, Dallol A, Al-Kattabi $H$, Chaudhary $A$, et al. Prognostic value of HER2 status in bladder transitional cell carcinoma revealed by both $\mathrm{IHC}$ and $\mathrm{BDISH}$ techniques. BMC Cancer. 2016; 16(1):653-61. 
25. Khalid El Gehani KJ, Al-Kikhia L, Emaetig F, Syrjanen $\mathrm{K}, \mathrm{Al}-$ Fituri O, Elzagheid A. Overexpression of HER2 is associated with the stage in carcinomas of the urinary bladder. Libyan J Medicine 2012; 7(1):32-39.

26. Ismail NEH, El-Nagar SI, Khodeir MM, Ahmed MM. Immunohistochemical Study of HER-2/neu Expression in Urothelial Bladder Carcinoma. Academic J Cancer Res. 2015; 8(2):35-9.

27. Jamal N, Hashmi SN, Jamal S. Evaluation of her$2 /$ neu expression by immunohistochemistry in urothelial carcinoma of urinary bladder. Pak Armed Forces Med J. 2015; 65(1):149-52.
DATA SHARING STATEMENT: The data that support the findings of this study are available on request from the corresponding author. The data are not publicly available due to privacy or ethical restrictions.

CONFLICT OF INTEREST: Authors declare no conflict of interest.

GRANTED SUPPORT AND FINANCIAL DISCLOSURE: Nil

\section{AUTHOR'S CONTRIBUTION}

Following authors have made substantial contributions to the manuscript as under

Bib M:

Concept and design of study, Collection of data, statistical analysis

Shah W, Asif M,: Writing of manuscript, critical review of manuscript

Khattak MT: Analysis and interpretation of data, statistical analysis

Jamil A, Nasib B: Data collection, bibliography

Authors agree to be accountable for all aspects of the work in ensuring that questions related to the accuracy or integrity of any part of the work are appropriately investigated and resolved. 